\title{
Multiple Novels and Accurate Traveling Wave and Numerical Solutions of the (2+1) Dimensional Fisher-Kolmogorov- Petrovskii-Piskunov Equation
}

\author{
Mostafa M. A. Khater ${ }^{1,2, *,+(\mathbb{D})}$ and Aliaa Mahfooz Alabdali ${ }^{3,+}$ \\ 1 Department of Mathematics, Faculty of Science, Jiangsu University, Zhenjiang 212013, China \\ 2 Department of Mathematics, Obour High Institute for Engineering and Technology, Cairo 11828, Egypt \\ 3 Faculty of Computing and information Technology Rabigh, King Abdulaziz University, Rabigh, \\ Makkah 21911, Saudi Arabia; amalabdaly@kau.edu.sa \\ * Correspondence: 1000005364@ujs.edu.cn \\ + These authors did all this work equally.
}

check for

updates

Citation: Khater, M.M.A.; Alabdali, A.M. Multiple Novels and Accurate Traveling Wave and Numerical

Solutions of the $(2+1)$ Dimensional Fisher-Kolmogorov-PetrovskiiPiskunov Equation. Mathematics 2021, 9, 1440. https://doi.org/10.3390/ math9121440

Academic Editor: José A. Tenreiro Machado

Received: 18 May 2021

Accepted: 18 June 2021

Published: 21 June 2021

Publisher's Note: MDPI stays neutral with regard to jurisdictional claims in published maps and institutional affiliations.

Copyright: (c) 2021 by the authors. Licensee MDPI, Basel, Switzerland. This article is an open access article distributed under the terms and conditions of the Creative Commons Attribution (CC BY) license (https:/ / creativecommons.org/licenses/by/ $4.0 /)$.

\begin{abstract}
The analytical and numerical solutions of the $(2+1)$ dimensional, Fisher-KolmogorovPetrovskii-Piskunov $((2+1)$ D-Fisher-KPP) model are investigated by employing the modified direct algebraic (MDA), modified Kudryashov (MKud.), and trigonometric-quantic B-spline (TQBS) schemes. This model, which arises in population genetics and nematic liquid crystals, describes the reaction-diffusion system by traveling waves in population genetics and the propagation of domain walls, pattern formation in bi-stable systems, and nematic liquid crystals. Many novel analytical solutions are constructed. These solutions are used to evaluate the requested numerical technique's conditions. The numerical solutions of the considered model are studied, and the absolute value of error between analytical and numerical is calculated to demonstrate the matching between both solutions. Some figures are represented to explain the obtained analytical solutions and the match between analytical and numerical results. The used schemes' performance shows their effectiveness and power and their ability to handle many nonlinear evolution equations.
\end{abstract}

Keywords: (2+1) D-Fisher-KPP model; computational and approximate solutions

\section{Introduction}

In the last century, and especially in the biological system, diffusion has been employed as one of the most famous models for spatial spread. It has been used for several services, such as invasion and pattern formation, ecology, motile cell populations, wound healing, the capillary growth network, the spatial movement of cell populations, and so on [1-5]. However, for studying closely packed cells such as epithelium [6], the linear diffusion model is not considered as an excellent idea where it contains a movement cell population; that is why the reaction-diffusion equation is a perfect bi-mathematical model [7]. Thus, the definition of the diffusion term in different cell populations is the ability of various cell populations to mix entirely. However, the fact shows in the different side where the cell will stop moving when it suddenly comes across another section that is well-known by contact inhibition of migration [8]. Thus, the wave propagation is considered as the ideal way of conveying biological information through the cells [9]. The simple model of the diffusion equation in one dimensional is given by [10-13]

$$
\mathcal{C}_{t}=\mathrm{Y} \mathcal{C}_{x x}
$$

where $\mathcal{C}=\mathcal{C}(x, t), \mathrm{Y}$ respectively describe the nutrient or cell concentration and diffusion coefficient, while the exchanging information's time is evaluated by $\mathcal{Q}\left(\frac{\mathcal{L}^{2}}{Y}\right)$, where $\mathcal{L}$ is the length of the domain. 
Fisher and Kolmogorov give the classical model for propagating an advantageous gene in a one-dimensional habitat [14]. This model is provided by $[15,16]$

$$
\mathcal{C}_{t}=\mathrm{Y} \mathcal{C}_{x x}+\zeta \mathcal{C}(1-\mathcal{C})
$$

where $\zeta$ demonstrates the growth rate of the chemical reaction. This model is known too with the extended Fisher-Kolmogorov equation [17] and the Fisher-KPP equation [18]. Equation (2) takes into account diffusion processes in accordance with Fick's law (term Y $\mathcal{C}_{x x}$ ), population reproduction at a rate $\zeta$ linear term $\zeta \mathcal{C}$ and density-dependent losses (term $\left.\zeta \mathcal{C}^{2}\right)$. This model has also been used in several fields, such as auto-catalytic chemical reactions, flame propagation, neurophysiology, logistic growth models, and the nuclear reactor theory. Luther was the first researcher who found the wave speed of this model [19]. Many researchers have investigated the traveling wave solutions of this model, and they have also improved it into (2+1)-dimensional form, that is given by [20-23]

$$
\mathcal{F}_{t}+\mu\left(\mathcal{F}_{x x}+2 \mathcal{F}_{x y}+\mathcal{F}_{y y}\right)-\left(\mathcal{F}_{x}+\mathcal{F}_{y}\right)+\Im(\mathcal{F})=0,
$$

where $\mu$ is a positive diffusion coefficient, while $\mathcal{F}=\mathcal{F}(x, y, t)$ is a function of the chemical concentration. Handling Equation (3) through the following wave transformation $\mathcal{F}(x, y, t)=\mathcal{K}(\mathfrak{S}), \mathfrak{S}=x+y+r t$, where $r$ is an arbitrary constant to be evaluated later, converts Equation (3) into the following nonlinear ordinary differential equation

$$
(r-2) \mathcal{K}^{\prime}+\mathcal{K}^{3}+4 \mu \mathcal{K}^{\prime \prime}-\mathcal{K}=0
$$

where $\left(\Im(\mathcal{F})=\mathcal{F}^{3}-\mathcal{F}\right)$ [17]. In this context, investigating this model is considered a primary icon in various fields [24-26]. Based on the computer revolution that has a significant effect on deriving computational, semi-analytical, and numerical schemes, many schemes have derived, such as the sech-tanh expansion method, auxiliary equation method, direct algebraic equation method, iteration method, exponential expansion method, Bspline schemes, Kudryashov methods, Adomian decomposition method, Khater methods, $\left(\frac{\Theta^{\prime}}{\Theta}\right)$-expansion methods, and so on [27-33]. This paper employs three recent analytical and numerical techniques [34-38] to investigate novel analytical wave solutions of Equation (3)'s accuracy of the used analytical schemes. colorred Our results in the case $n=1$ are proved using the homogeneous balance principles and the well-known frameworks of the MDA and MKud. methods. Thus, the general solutions of Equation (4) are evaluated by $[39,40]$

$$
\mathcal{K}(\mathfrak{S})=\left\{\begin{array}{c}
\sum_{i=-n}^{n} a_{i} \mathfrak{Z}(\mathfrak{S})^{i}=a_{1} \mathfrak{Z}(\mathfrak{S})+\frac{a_{-1}}{\mathfrak{Z}(\mathfrak{S})}+a_{0} \\
\sum_{i=0}^{n} a_{i} \mathfrak{G}(\mathfrak{S})^{i}=a_{1} \mathfrak{G}(\mathfrak{S})+a_{0},
\end{array}\right.
$$

where $a_{0}, a_{1}, a_{-1}$ are arbitrary constants.

The other sections in this paper are given in the following order; Section 2 shows the novel and accurate solutions of the considered model through the above-mentioned analytical and numerical schemes. Section 3 demonstrates the novelty and originality of our paper. Section 4 gives a summary of the manuscript.

\section{Analytical and Numerical Matching for the (2+1) D-Fisher-KPP Model}

Here, the matching between the analytical and numerical solutions is discussed through implementing two recent analytical (MDA and MKud.) and one numerical (TQBS) scheme to Equation (4). This study also shows the accuracy of the obtained computational solutions and strategies used. 


\subsection{MDA Analytical Versus TQBS Numerical Techniques along (2+1) D-Fisher-KPP Model}

Applying the MDA scheme's framework and its auxiliary $\left(\mathrm{Y}^{\prime}(\mathfrak{S})=l_{3} \mathrm{Y}(\mathfrak{S})^{2}+l_{2} \mathrm{Y}(\mathfrak{S})\right.$ $\left.+l_{1}\right)$ where $l_{i}, i=1,2,3$ are arbitrary constants to be determined later, to Equation (4), we obtain the following sets of the above-mentioned parameters:

Set I

$$
\begin{aligned}
& a_{0}=\frac{\sqrt{\left(l_{2}^{2}-4 l_{1} l_{3}\right)^{2}}-l_{2} \sqrt{l_{2}^{2}-4 l_{1} l_{3}}}{2\left(l_{2}^{2}-4 l_{1} l_{3}\right)}, a_{-1}=-\frac{l_{1}}{\sqrt{l_{2}^{2}-4 l_{1} l_{3}}}, a_{1}=0, r=2-\frac{3 \sqrt{\left(l_{2}^{2}-4 l_{1} l_{3}\right)^{2}}}{2\left(l_{2}^{2}-4 l_{1} l_{3}\right)^{3 / 2}}, \\
& \mu=-\frac{1}{4\left(l_{2}^{2}-4 l_{1} l_{3}\right)} .
\end{aligned}
$$

\section{Set II}

$$
\begin{aligned}
& a_{0}=\frac{\sqrt{\left(l_{2}^{2}-4 l_{1} l_{3}\right)^{2}}-l_{2} \sqrt{l_{2}^{2}-4 l_{1} l_{3}}}{2\left(l_{2}^{2}-4 l_{1} l_{3}\right)}, a_{-1}=0, a_{1}=-\frac{l_{3}}{\sqrt{l_{2}^{2}-4 l_{1} l_{3}}}, r=\frac{3 \sqrt{\left(l_{2}^{2}-4 l_{1} l_{3}\right)^{2}}}{2\left(l_{2}^{2}-4 l_{1} l_{3}\right)^{3 / 2}}+2, \\
& \mu=-\frac{1}{4\left(l_{2}^{2}-4 l_{1} l_{3}\right)} .
\end{aligned}
$$

\section{Set III}

$$
a_{0}=\frac{l_{2}}{\sqrt{l_{2}^{2}-4 l_{1} l_{3}}}, a_{-1}=0, a_{1}=\frac{2 l_{3}}{\sqrt{l_{2}^{2}-4 l_{1} l_{3}}}, r=2, \mu=\frac{1}{4 l_{1} l_{3}-l_{2}^{2}} .
$$

Therefore, the computational wave solutions of the $(2+1) \mathrm{D}-(2+1)$ D-Fisher-KPP model are constructed in the following formulas:

For $l_{2}=0, l_{1} l_{3}>0$, we get

$$
\begin{gathered}
\mathcal{F}_{\mathrm{I}, 1}(x, y, t)=\frac{\sqrt{-l_{1} l_{3}} \sqrt{l_{1} l_{3}}}{2 l_{1} l_{3}} \cot \left(\sqrt{l_{1} l_{3}}\left(\eta-\frac{3 \sqrt{l_{1}^{2} l_{3}^{2}} t}{4\left(-l_{1} l_{3}\right)^{3 / 2}}+2 t+x+y\right)\right)-\frac{\sqrt{l_{1}^{2} l_{3}^{2}}}{2 l_{1} l_{3}}, \\
\mathcal{F}_{\mathrm{I}, 2}(x, y, t)=\frac{\sqrt{-l_{1} l_{3}} \sqrt{l_{1} l_{3}}}{2 l_{1} l_{3}} \tan \left(\sqrt{l_{1} l_{3}}\left(\eta-\frac{3 \sqrt{l_{1}^{2} l_{3}^{2}} t}{4\left(-l_{1} l_{3}\right)^{3 / 2}}+2 t+x+y\right)\right)-\frac{\sqrt{l_{1}^{2} l_{3}^{2}}}{2 l_{1} l_{3}}, \\
\mathcal{F}_{\mathrm{II}, 1}(x, y, t)=\frac{\sqrt{-l_{1} l_{3}} \sqrt{l_{1} l_{3}}}{2 l_{1} l_{3}} \tan \left(\sqrt{l_{1} l_{3}}\left(\eta+\frac{3 \sqrt{l_{1}^{2} l_{3}^{2}} t}{4\left(-l_{1} l_{3}\right)^{3 / 2}}+2 t+x+y\right)\right)-\frac{\sqrt{l_{1}^{2} l_{3}^{2}}}{2 l_{1} l_{3}}, \\
\mathcal{F}_{\mathrm{II}, 2}(x, y, t)=\frac{\sqrt{-l_{1} l_{3}} \sqrt{l_{1} l_{3}}}{2 l_{1} l_{3}} \cot \left(\sqrt{l_{1} l_{3}}\left(\eta+\frac{3 \sqrt{l_{1}^{2} l_{3}^{2}} t}{4\left(-l_{1} l_{3}\right)^{3 / 2}}+2 t+x+y\right)\right)-\frac{\sqrt{l_{1}^{2} l_{3}^{2}}}{2 l_{1} l_{3}}, \\
\mathcal{F}_{\mathrm{III}, 1}(x, y, t)=-\frac{\sqrt{-l_{1} l_{3}} \sqrt{l_{1} l_{3}}}{l_{1} l_{3}} \tan \left(\sqrt{l_{1} l_{3}}(\eta+2 t+x+y)\right),
\end{gathered}
$$




$$
\mathcal{F}_{\mathrm{III}, 2}(x, y, t)=-\frac{\sqrt{-l_{1} l_{3}} \sqrt{l_{1} l_{3}}}{l_{1} l_{3}} \cot \left(\sqrt{l_{1} l_{3}}(\eta+2 t+x+y)\right) .
$$

For $l_{2}=0, l_{1} l_{3}<0$, we get

$$
\begin{aligned}
\mathcal{F}_{\mathrm{I}, 3}(x, y, t)= & \frac{1}{2} \operatorname{coth}\left(\sqrt{-l_{1} l_{3}}\left(-\frac{3 \sqrt{l_{1}^{2} l_{3}^{2}} t}{4\left(-l_{1} l_{3}\right)^{3 / 2}}+2 t+x+y\right) \mp \frac{\log (\eta)}{2}\right)-\frac{\sqrt{l_{1}^{2} l_{3}^{2}}}{2 l_{1} l_{3}}, \\
\mathcal{F}_{\mathrm{I}, 4}(x, y, t)= & \frac{1}{2} \tanh \left(\sqrt{-l_{1} l_{3}}\left(-\frac{3 \sqrt{l_{1}^{2} l_{3}^{2}} t}{4\left(-l_{1} l_{3}\right)^{3 / 2}}+2 t+x+y\right) \mp \frac{\log (\eta)}{2}\right)-\frac{\sqrt{l_{1}^{2} l_{3}^{2}}}{2 l_{1} l_{3}}, \\
\mathcal{F}_{\mathrm{II}, 3}(x, y, t)= & -\frac{1}{2} \tanh \left(\sqrt{-l_{1} l_{3}}\left(\frac{3 \sqrt{l_{1}^{2} l_{3}^{2}} t}{4\left(-l_{1} l_{3}\right)^{3 / 2}}+2 t+x+y\right) \mp \frac{\log (\eta)}{2}\right)-\frac{\sqrt{l_{1}^{2} l_{3}^{2}}}{2 l_{1} l_{3}}, \\
\mathcal{F}_{\mathrm{II}, 4}(x, y, t)= & -\frac{1}{2} \operatorname{coth}\left(\sqrt{-l_{1} l_{3}}\left(\frac{3 \sqrt{l_{1}^{2} l_{3}^{2}} t}{4\left(-l_{1} l_{3}\right)^{3 / 2}}+2 t+x+y\right) \mp \frac{\log (\eta)}{2}\right)-\frac{\sqrt{l_{1}^{2} l_{3}^{2}}}{2 l_{1} l_{3}}, \\
& \mathcal{F}_{\mathrm{III}, 3}(x, y, t)=\tanh \left(\sqrt{-l_{1} l_{3}}(2 t+x+y) \mp \frac{\log (\eta)}{2}\right), \\
& \mathcal{F}_{\mathrm{III}, 4}(x, y, t)=\operatorname{coth}\left(\sqrt{-l_{1} l_{3}}(2 t+x+y) \mp \frac{\log (\eta)}{2}\right) .
\end{aligned}
$$

For $l_{1}=0, l_{2}>0$, we get

$$
\begin{gathered}
\mathcal{F}_{\mathrm{II}, 5}(x, y, t)=\frac{\sqrt{l_{2}^{2}}}{l_{2}\left(l_{3} \exp \left(l_{2}\left(\eta+\frac{3 \sqrt{l_{2}^{4}} t}{2\left(l_{2}^{2}\right)^{3 / 2}}+2 t+x+y\right)\right)-1\right)}+\frac{\sqrt{l_{2}^{2}}}{2 l_{2}}+\frac{\sqrt{l_{2}^{4}}}{2 l_{2}^{2}}, \\
\mathcal{F}_{\mathrm{III}, 5}(x, y, t)=-\frac{l_{3} \sqrt{l_{2}^{2}} e^{l_{2}(\eta+2 t+x+y)}}{l_{2}\left(l_{3} e^{l_{2}(\eta+2 t+x+y)}-1\right)}-\frac{\sqrt{l_{2}^{2}}}{l_{2}\left(l_{3} e^{l_{2}(\eta+2 t+x+y)}-1\right)} .
\end{gathered}
$$

For $l_{1}=0, l_{2}<0$, we get

$$
\begin{array}{r}
\mathcal{F}_{\mathrm{II}, 6}(x, y, t)=-\frac{l_{3}}{\sqrt{l_{2}^{2}}\left(l_{3} \exp \left(l_{2}\left(\eta+\frac{3 \sqrt{l_{2}^{4}} t}{2\left(l_{2}^{2}\right)^{3 / 2}}+2 t+x+y\right)\right)+1\right)}-\frac{\sqrt{l_{2}^{2}}}{2 l_{2}}+\frac{\sqrt{l_{2}^{4}}}{2 l_{2}^{2}}+\frac{l_{3}}{\sqrt{l_{2}^{2}}}, \\
\mathcal{F}_{\mathrm{III}, 6}(x, y, t)=\frac{2 l_{3}}{\sqrt{l_{2}^{2}}\left(l_{3} e^{l_{2}(\eta+2 t+x+y)}+1\right)}+\frac{\sqrt{l_{2}^{2}}}{l_{2}}-\frac{2 l_{3}}{\sqrt{l_{2}^{2}}} .
\end{array}
$$


For $4 l_{1} l_{3}>l_{2}^{2}$, we get

$$
\begin{aligned}
& \mathcal{F}_{\mathrm{I}, 5}(x, y, t)=\frac{2 l_{1} l_{3}}{\sqrt{l_{2}^{2}-4 l_{1} l_{3}}\left(l_{2}-\sqrt{4 l_{1} l_{3}-l_{2}^{2}} \tan \left(\frac{1}{2} \sqrt{4 l_{1} l_{3}-l_{2}^{2}}\left(\eta-\frac{3 \sqrt{\left(l_{2}^{2}-4 l_{1} l_{3}\right)^{2}} t}{2\left(l_{2}^{2}-4 l_{1} l_{3}\right)^{3 / 2}}+2 t+x+y\right)\right)\right)} \\
& +\frac{l_{2}^{2}}{2 \sqrt{\left(l_{2}^{2}-4 l_{1} l_{3}\right)^{2}}}-\frac{l_{2}}{2 \sqrt{l_{2}^{2}-4 l_{1} l_{3}}}-\frac{2 l_{1} l_{3}}{\sqrt{\left(l_{2}^{2}-4 l_{1} l_{3}\right)^{2}}} \\
& \mathcal{F}_{\mathrm{I}, 6}(x, y, t)=\frac{2 l_{1} l_{3}}{\sqrt{l_{2}^{2}-4 l_{1} l_{3}}\left(l_{2}-\sqrt{4 l_{1} l_{3}-l_{2}^{2}} \cot \left(\frac{1}{2} \sqrt{4 l_{1} l_{3}-l_{2}^{2}}\left(\eta-\frac{3 \sqrt{\left(l_{2}^{2}-4 l_{1} l_{3}\right)^{2}} t}{2\left(l_{2}^{2}-4 l_{1} l_{3}\right)^{3 / 2}}+2 t+x+y\right)\right)\right)} \\
& +\frac{l_{2}^{2}}{2 \sqrt{\left(l_{2}^{2}-4 l_{1} l_{3}\right)^{2}}}-\frac{l_{2}}{2 \sqrt{l_{2}^{2}-4 l_{1} l_{3}}}-\frac{2 l_{1} l_{3}}{\sqrt{\left(l_{2}^{2}-4 l_{1} l_{3}\right)^{2}}} \\
& \mathcal{F}_{\mathrm{II}, 7}(x, y, t)=-\frac{\sqrt{-\left(l_{2}^{2}-4 l_{1} l_{3}\right)^{2}} \tan \left(\frac{1}{2} \sqrt{4 l_{1} l_{3}-l_{2}^{2}}\left(\eta+\frac{3 \sqrt{\left(l_{2}^{2}-4 l_{1} l_{3}\right)^{2}} t}{2\left(l_{2}^{2}-4 l_{1} l_{3}\right)^{3 / 2}}+2 t+x+y\right)\right)}{2\left(l_{2}^{2}-4 l_{1} l_{3}\right)} \\
& +\frac{\sqrt{\left(l_{2}^{2}-4 l_{1} l_{3}\right)^{2}}}{2\left(l_{2}^{2}-4 l_{1} l_{3}\right)} \\
& \mathcal{F}_{\mathrm{II}, 8}(x, y, t)=-\frac{\sqrt{-\left(l_{2}^{2}-4 l_{1} l_{3}\right)^{2}} \cot \left(\frac{1}{2} \sqrt{4 l_{1} l_{3}-l_{2}^{2}}\left(\eta+\frac{3 \sqrt{\left(l_{2}^{2}-4 l_{1} l_{3}\right)^{2}} t}{2\left(l_{2}^{2}-4 l_{1} l_{3}\right)^{3 / 2}}+2 t+x+y\right)\right)}{2\left(l_{2}^{2}-4 l_{1} l_{3}\right)} \\
& +\frac{\sqrt{\left(l_{2}^{2}-4 l_{1} l_{3}\right)^{2}}}{2\left(l_{2}^{2}-4 l_{1} l_{3}\right)} \\
& \mathcal{F}_{\text {III, },}(x, y, t)=\frac{\sqrt{4 l_{1} l_{3}-l_{2}^{2}}}{\sqrt{l_{2}^{2}-4 l_{1} l_{3}}} \tan \left(\frac{1}{2} \sqrt{4 l_{1} l_{3}-l_{2}^{2}}(\eta+2 t+x+y)\right), \\
& \mathcal{F}_{\mathrm{III}, 8}(x, y, t)=\frac{\sqrt{4 l_{1} l_{3}-l_{2}^{2}}}{\sqrt{l_{2}^{2}-4 l_{1} l_{3}}} \cot \left(\frac{1}{2} \sqrt{4 l_{1} l_{3}-l_{2}^{2}}(\eta+2 t+x+y)\right) .
\end{aligned}
$$

Matching between Analytical and Numerical

Applying the TQBS numerical technique with the following initial condition $\mathcal{F}(x, 0,0)=\frac{1}{2} \tanh (25 x)+\frac{1}{2}$ gets the following shown analytical and numerical solutions' values with respect to different values of $x$ in Table 1 . 
Table 1. Analytical and numerical matching through MDA and TQBS schemes.

\begin{tabular}{|c|c|c|c|c|c|c|c|}
\hline Value of $x$ & Analytical & Numerical & Error & Value of $x$ & Analytical & Numerical & Error \\
\hline 0 & 0.5 & 0.5 & 0 & 0.515625 & 0.925947 & 0.999254 & 0.073307 \\
\hline 0.015625 & 0.519127 & 0.596515 & 0.077388 & 0.53125 & 0.931028 & 0.999203 & 0.068176 \\
\hline 0.03125 & 0.538199 & 0.705602 & 0.167403 & 0.546875 & 0.935784 & 0.999146 & 0.063362 \\
\hline 0.046875 & 0.557159 & 0.77828 & 0.221121 & 0.5625 & 0.940233 & 0.999082 & 0.058849 \\
\hline 0.0625 & 0.575954 & 0.835791 & 0.259837 & 0.578125 & 0.944392 & 0.999013 & 0.05462 \\
\hline 0.078125 & 0.594532 & 0.878033 & 0.283501 & 0.59375 & 0.948278 & 0.998936 & 0.050658 \\
\hline 0.09375 & 0.612843 & 0.909745 & 0.296901 & 0.609375 & 0.951906 & 0.998854 & 0.046947 \\
\hline 0.109375 & 0.630841 & 0.933241 & 0.3024 & 0.625 & 0.955292 & 0.998764 & 0.043472 \\
\hline 0.125 & 0.648482 & 0.950674 & 0.302192 & 0.640625 & 0.95845 & 0.998667 & 0.040217 \\
\hline 0.140625 & 0.665726 & 0.963567 & 0.297841 & 0.65625 & 0.961393 & 0.998561 & 0.037168 \\
\hline 0.15625 & 0.682539 & 0.973094 & 0.290555 & 0.671875 & 0.964136 & 0.998448 & 0.034312 \\
\hline 0.171875 & 0.698889 & 0.980124 & 0.281235 & 0.6875 & 0.966691 & 0.998325 & 0.031634 \\
\hline 0.1875 & 0.714748 & 0.985307 & 0.270559 & 0.703125 & 0.96907 & 0.998192 & 0.029123 \\
\hline 0.203125 & 0.730095 & 0.989125 & 0.25903 & 0.71875 & 0.971283 & 0.998049 & 0.026765 \\
\hline 0.21875 & 0.744911 & 0.991936 & 0.247025 & 0.734375 & 0.973343 & 0.997894 & 0.024551 \\
\hline 0.234375 & 0.759182 & 0.994003 & 0.234821 & 0.75 & 0.975259 & 0.997727 & 0.022468 \\
\hline 0.25 & 0.772897 & 0.995521 & 0.222623 & 0.765625 & 0.97704 & 0.997547 & 0.020506 \\
\hline 0.265625 & 0.786052 & 0.996634 & 0.210582 & 0.78125 & 0.978696 & 0.997352 & 0.018656 \\
\hline 0.28125 & 0.798644 & 0.997448 & 0.198805 & 0.796875 & 0.980235 & 0.997142 & 0.016907 \\
\hline 0.296875 & 0.810672 & 0.998042 & 0.18737 & 0.8125 & 0.981665 & 0.996915 & 0.015251 \\
\hline 0.3125 & 0.822143 & 0.998474 & 0.176331 & 0.828125 & 0.982993 & 0.996671 & 0.013678 \\
\hline 0.328125 & 0.833061 & 0.998784 & 0.165724 & 0.84375 & 0.984226 & 0.996407 & 0.012181 \\
\hline 0.34375 & 0.843437 & 0.999006 & 0.155569 & 0.859375 & 0.985372 & 0.996122 & 0.010751 \\
\hline 0.359375 & 0.853281 & 0.999161 & 0.14588 & 0.875 & 0.986435 & 0.995815 & 0.00938 \\
\hline 0.375 & 0.862607 & 0.999267 & 0.13666 & 0.890625 & 0.987422 & 0.995484 & 0.008062 \\
\hline 0.390625 & 0.87143 & 0.999336 & 0.127906 & 0.90625 & 0.988338 & 0.995126 & 0.006788 \\
\hline 0.40625 & 0.879765 & 0.999377 & 0.119611 & 0.921875 & 0.989188 & 0.99474 & 0.005552 \\
\hline 0.421875 & 0.88763 & 0.999396 & 0.111766 & 0.9375 & 0.989977 & 0.994326 & 0.004349 \\
\hline 0.4375 & 0.895041 & 0.999399 & 0.104357 & 0.953125 & 0.990709 & 0.993871 & 0.003162 \\
\hline 0.453125 & 0.902018 & 0.999388 & 0.09737 & 0.96875 & 0.991387 & 0.993411 & 0.002023 \\
\hline 0.46875 & 0.908578 & 0.999367 & 0.090789 & 0.984375 & 0.992017 & 0.992795 & 0.000778 \\
\hline 0.484375 & 0.914741 & 0.999337 & 0.084596 & 1 & 0.992601 & 0.992601 & $5.55 \times 10^{-17}$ \\
\hline
\end{tabular}

2.2. Kud Analytical vs. TQBS Numerical Techniques along (2+1) D-Fisher-KPP Model

Applying the MKud. scheme's framework and its auxiliary $\left(\Gamma^{\prime}(\mathfrak{S})=\ln (k)\left(\Gamma(\mathfrak{S})^{2}\right.\right.$ $-\Gamma(\mathfrak{S}))$ where $k$ is an arbitrary constant to be determined later, to Equation (4), we obtain the following sets of the above-mentioned parameters:

Set I

$$
a_{0}=-1, a_{1}=2, r=2, \mu=-\frac{1}{\log ^{2}(k)}
$$




$$
a_{0}=0, a_{1}=1, r=\frac{4 \log (k)-3}{2 \log (k)}, \mu=-\frac{1}{4 \log ^{2}(k)} .
$$

\section{Set III}

$$
a_{0}=1, a_{1}=-1, r=\frac{4 \log (k)+3}{2 \log (k)}, \mu=-\frac{1}{4 \log ^{2}(k)} .
$$

Therefore, the computational wave solutions of the (2+1) D-Fisher-KPP model are constructed in the following formulas:

$$
\begin{gathered}
\mathcal{S}_{\mathrm{I}}(x, y, t)=\frac{2}{1 \pm k^{2 t+x+y}}-1, \\
\mathcal{S}_{\mathrm{II}}(x, y, t)=\frac{1}{1 \pm k^{-\frac{3 t}{2 \log (k)}+2 t+x+y}}, \\
\mathcal{S}_{\mathrm{III}}(x, y, t)=1-\frac{1}{1 \pm k^{\frac{3 t}{2 \log (k)}+2 t+x+y}} .
\end{gathered}
$$

Semi-Analytical Solutions

Applying the TQBS numerical technique with the following initial condition $\mathcal{F}(x, 0,0)=\frac{1}{e^{x}+1}$ gets the following shown analytical and numerical solutions' values with respect to different values of $x$ in Table 2 .

Table 2. Analytical and numerical matching along MKud. and TQBS techniques.

\begin{tabular}{cccccccc}
\hline Value of $x$ & Analytical & Numerical & Error & Value of $x$ & Analytical & Numerical & Error \\
\hline 0 & 0.5 & 0.5 & 0 & 0.515625 & 0.373876 & 0.373876 & $1.62 \times 10^{-11}$ \\
0.015625 & 0.496094 & 0.496094 & $1.2 \times 10^{-12}$ & 0.53125 & 0.370225 & 0.370225 & $1.59 \times 10^{-11}$ \\
0.03125 & 0.492188 & 0.492188 & $2.94 \times 10^{-12}$ & 0.546875 & 0.36659 & 0.36659 & $1.56 \times 10^{-11}$ \\
0.046875 & 0.488283 & 0.488283 & $4.39 \times 10^{-12}$ & 0.5625 & 0.362969 & 0.362969 & $1.52 \times 10^{-11}$ \\
0.0625 & 0.48438 & 0.48438 & $5.79 \times 10^{-12}$ & 0.578125 & 0.359364 & 0.359364 & $1.49 \times 10^{-11}$ \\
0.078125 & 0.480479 & 0.480479 & $7.07 \times 10^{-12}$ & 0.59375 & 0.355775 & 0.355775 & $1.45 \times 10^{-11}$ \\
0.09375 & 0.47658 & 0.47658 & $8.26 \times 10^{-12}$ & 0.609375 & 0.352202 & 0.352202 & $1.41 \times 10^{-11}$ \\
0.109375 & 0.472683 & 0.472683 & $9.37 \times 10^{-12}$ & 0.625 & 0.348645 & 0.348645 & $1.37 \times 10^{-11}$ \\
0.125 & 0.468791 & 0.468791 & $1.04 \times 10^{-11}$ & 0.640625 & 0.345105 & 0.345105 & $1.32 \times 10^{-11}$ \\
0.140625 & 0.464902 & 0.464902 & $1.13 \times 10^{-11}$ & 0.65625 & 0.341582 & 0.341582 & $1.28 \times 10^{-11}$ \\
0.15625 & 0.461017 & 0.461017 & $1.22 \times 10^{-11}$ & 0.671875 & 0.338077 & 0.338077 & $1.23 \times 10^{-11}$ \\
0.171875 & 0.457137 & 0.457137 & $1.29 \times 10^{-11}$ & 0.6875 & 0.334589 & 0.334589 & $1.18 \times 10^{-11}$ \\
0.1875 & 0.453262 & 0.453262 & $1.36 \times 10^{-11}$ & 0.703125 & 0.33112 & 0.33112 & $1.13 \times 10^{-11}$ \\
0.203125 & 0.449393 & 0.449393 & $1.43 \times 10^{-11}$ & 0.71875 & 0.327668 & 0.327668 & $1.08 \times 10^{-11}$ \\
0.21875 & 0.44553 & 0.44553 & $1.48 \times 10^{-11}$ & 0.734375 & 0.324235 & 0.324235 & $1.02 \times 10^{-11}$ \\
0.234375 & 0.441673 & 0.441673 & $1.53 \times 10^{-11}$ & 0.75 & 0.320821 & 0.320821 & $9.68 \times 10^{-12}$ \\
0.25 & 0.437823 & 0.437823 & $1.58 \times 10^{-11}$ & 0.765625 & 0.317426 & 0.317426 & $9.13 \times 10^{-12}$ \\
0.265625 & 0.433981 & 0.433981 & $1.62 \times 10^{-11}$ & 0.78125 & 0.314051 & 0.314051 & $8.56 \times 10^{-12}$ \\
0.28125 & 0.430147 & 0.430147 & $1.65 \times 10^{-11}$ & 0.796875 & 0.310694 & 0.310694 & $7.98 \times 10^{-12}$ \\
0.296875 & 0.426322 & 0.426322 & $1.68 \times 10^{-11}$ & 0.8125 & 0.307358 & 0.307358 & $7.39 \times 10^{-12}$ \\
0.3125 & 0.422505 & 0.422505 & $1.7 \times 10^{-11}$ & 0.828125 & 0.304042 & 0.304042 & $6.8 \times 10^{-12}$ \\
0.328125 & 0.418697 & 0.418697 & $1.72 \times 10^{-11}$ & 0.84375 & 0.300746 & 0.300746 & $6.19 \times 10^{-12}$ \\
\hline & & & & & & &
\end{tabular}


Table 2. Cont.

\begin{tabular}{cccccccc}
\hline Value of $x$ & Analytical & Numerical & Error & Value of $x$ & Analytical & Numerical & Error \\
\hline 0.34375 & 0.414899 & 0.414899 & $1.73 \times 10^{-11}$ & 0.859375 & 0.29747 & 0.29747 & $5.58 \times 10^{-12}$ \\
0.359375 & 0.411111 & 0.411111 & $1.74 \times 10^{-11}$ & 0.875 & 0.294215 & 0.294215 & $4.96 \times 10^{-12}$ \\
0.375 & 0.407333 & 0.407333 & $1.74 \times 10^{-11}$ & 0.890625 & 0.290981 & 0.290981 & $4.34 \times 10^{-12}$ \\
0.390625 & 0.403567 & 0.403567 & $1.74 \times 10^{-11}$ & 0.90625 & 0.287768 & 0.287768 & $3.71 \times 10^{-12}$ \\
0.40625 & 0.399812 & 0.399812 & $1.74 \times 10^{-11}$ & 0.921875 & 0.284576 & 0.284576 & $3.07 \times 10^{-12}$ \\
0.421875 & 0.396068 & 0.396068 & $1.73 \times 10^{-11}$ & 0.9375 & 0.281406 & 0.281406 & $2.44 \times 10^{-12}$ \\
0.4375 & 0.392337 & 0.392337 & $1.72 \times 10^{-11}$ & 0.953125 & 0.278257 & 0.278257 & $1.79 \times 10^{-12}$ \\
0.453125 & 0.388618 & 0.388618 & $1.71 \times 10^{-11}$ & 0.96875 & 0.27513 & 0.27513 & $1.16 \times 10^{-12}$ \\
0.46875 & 0.384912 & 0.384912 & $1.69 \times 10^{-11}$ & 0.984375 & 0.272025 & 0.272025 & $4.57 \times 10^{-13}$ \\
0.484375 & 0.38122 & 0.38122 & $1.67 \times 10^{-11}$ & 1 & 0.268941 & 0.268941 & $5.55 \times 10^{-17}$ \\
\hline
\end{tabular}

\section{Results' Interpretation}

This section studies the originality and novelty of this research paper. It also shows the accuracy of the obtained analytical solutions. The MDA and MKud. computational schemes have been applied to the (2+1) D-Fisher-KPP model for constructed novel analytical wave solutions. Many distinct analytical wave solutions have been obtained, and some of them have been demonstrated through sketches in 2D, 3D, and contour plots. These figures explain the dynamical characterization of the reaction-diffusion system by traveling waves in population genetics and propagating domain walls and pattern formation in bi-stable systems in nematic liquid crystals. Figures 1-4 show some novel analytical wave solutions with different values to the above-mentioned parameters. Comparing our solutions with those that have been obtained in a previously published paper [17,41] shows our solutions are completely different from those that have been evaluated in [41]. Still, some of our solutions match the obtained solutions in [17] when $\alpha=1$, where in that paper, the authors in that paper studied the fractional form of the considered model.
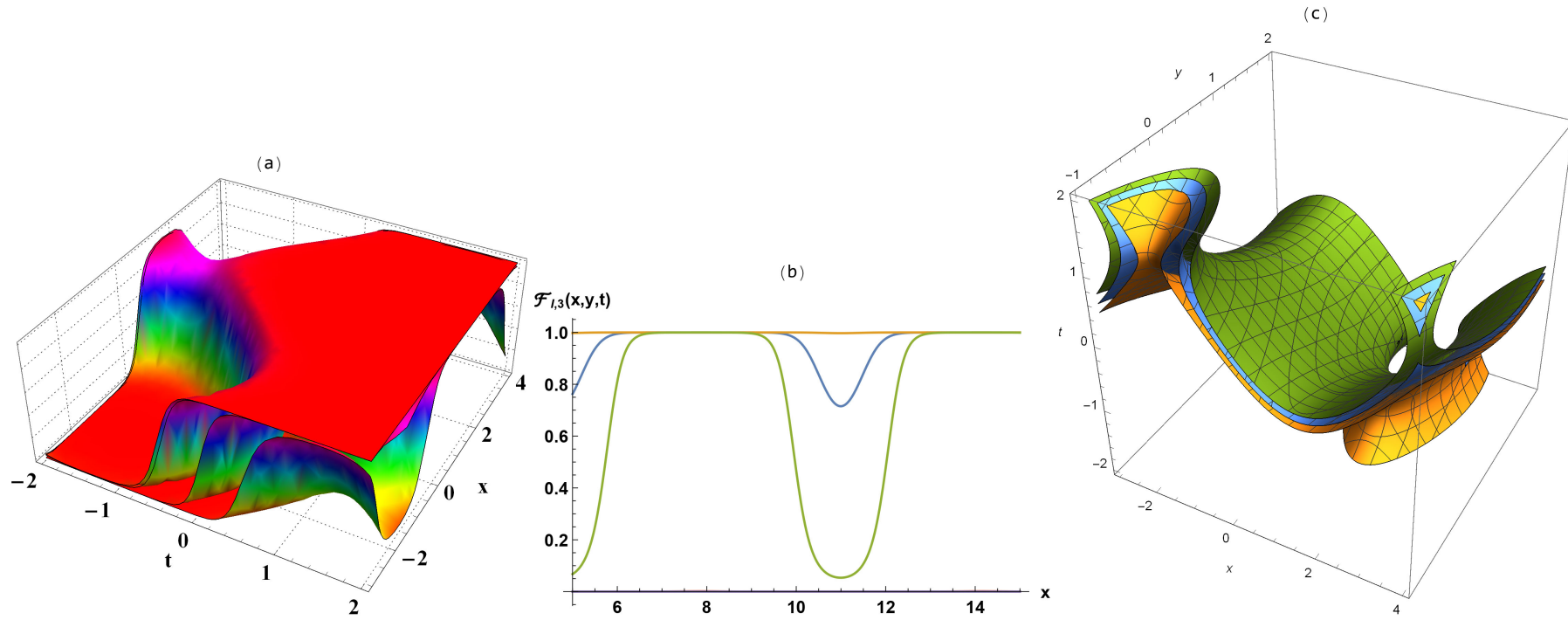

Figure 1. Computation wave solutions of Equation (12) in 3D (a), 2D (b), and contour (c) plots. 

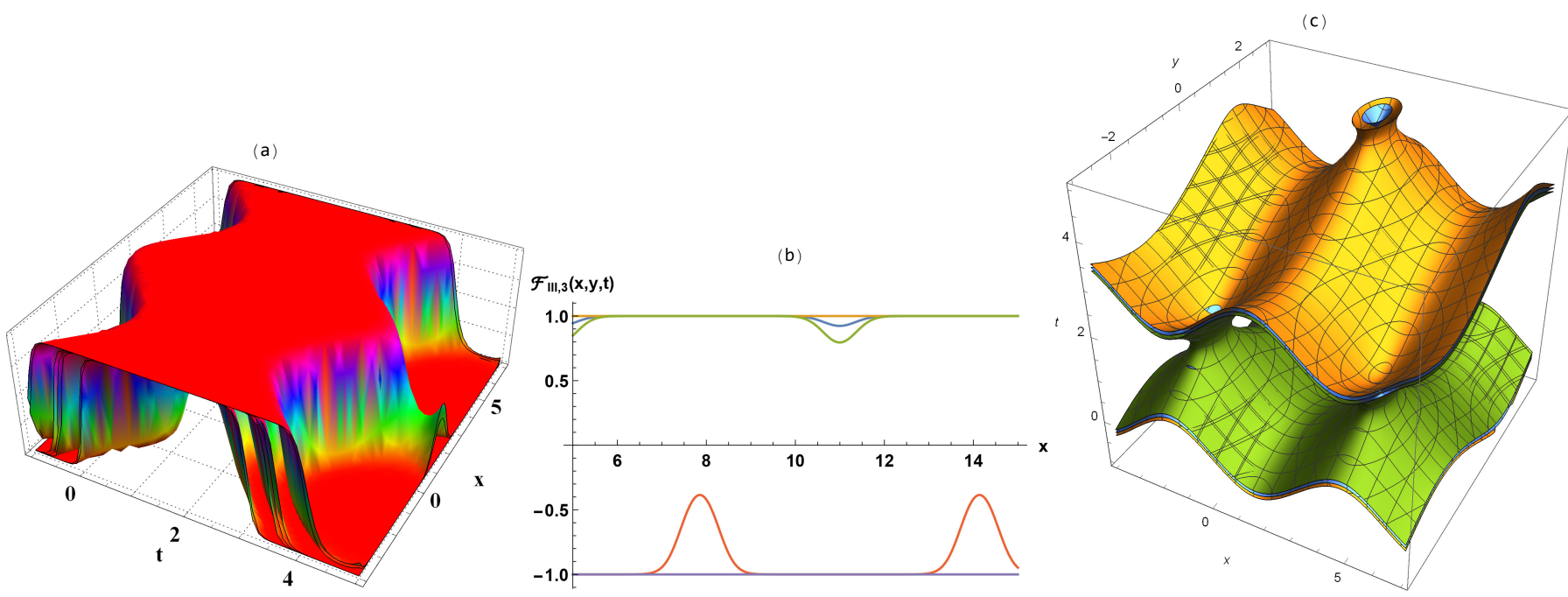

Figure 2. Computational wave solutions of Equation (16) in $3 \mathrm{D}(\mathbf{a}), 2 \mathrm{D}(\mathbf{b})$, and contour (c) plots.
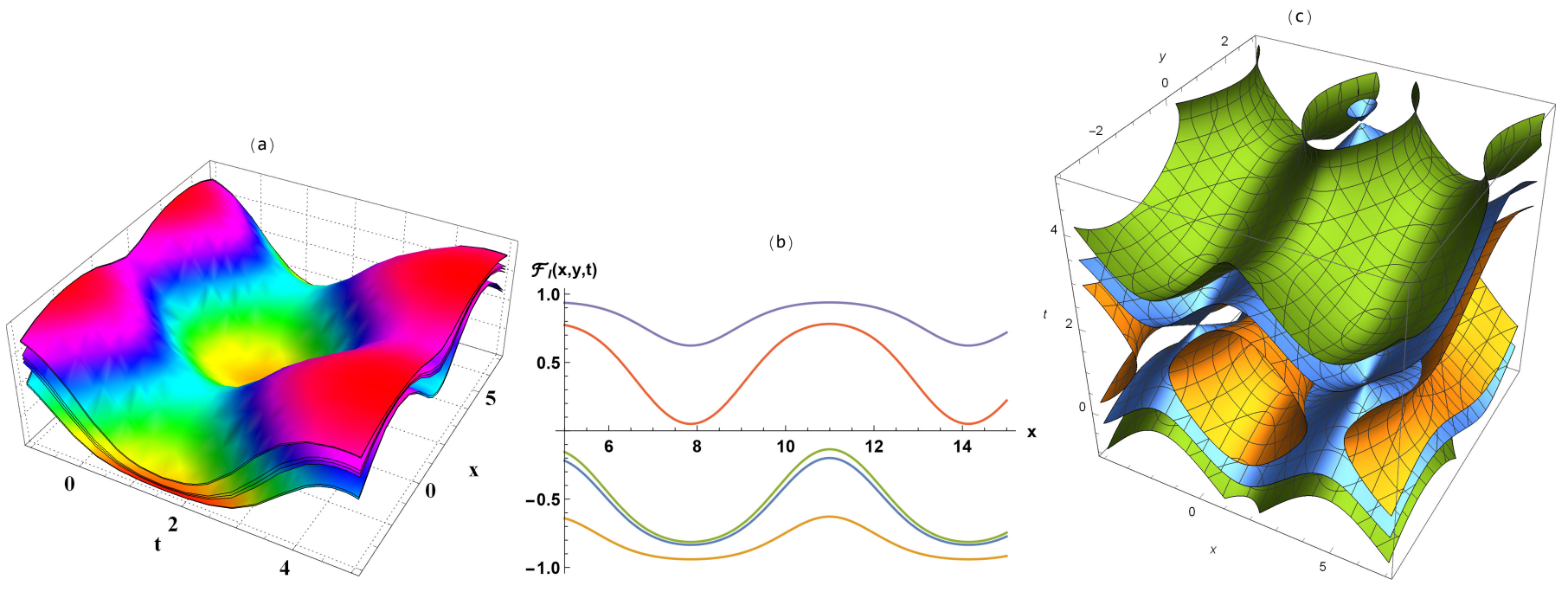

Figure 3. Computational wave solutions of Equation (28) in 3D (a), 2D (b), and contour (c) plots.

The TQBS scheme has been applied to the considered model based on the obtained computational solutions. The absolute error between the analytical and numerical solutions has been calculated to show the accuracy of the obtained results and used methods (Tables 1 and 2 and Figures 5 and 6). This calculating shows the arrogance of the MKud. method over the MDA method, where its absolute values of error are much smaller than those obtained by the MDA method (Figure 7). 

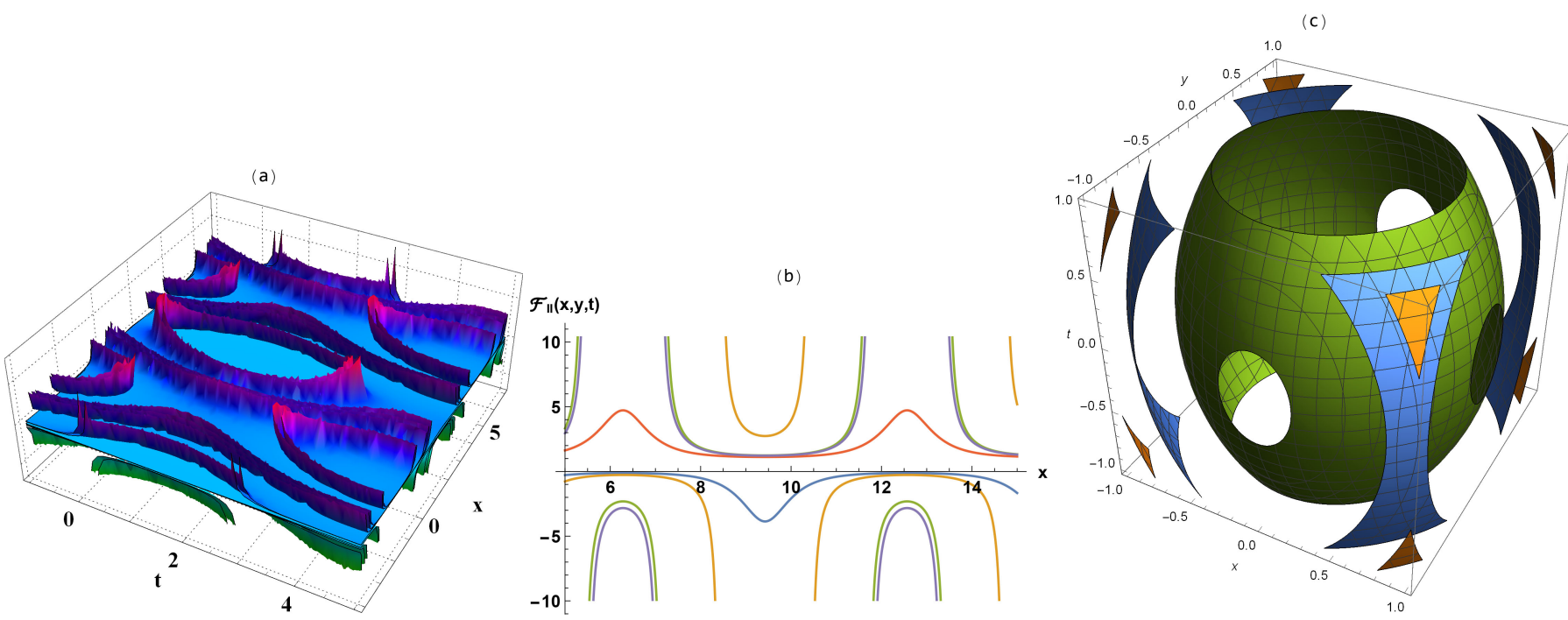

Figure 4. Computational wave solutions of Equation (29) in 3D (a), 2D (b), and contour (c) plots.
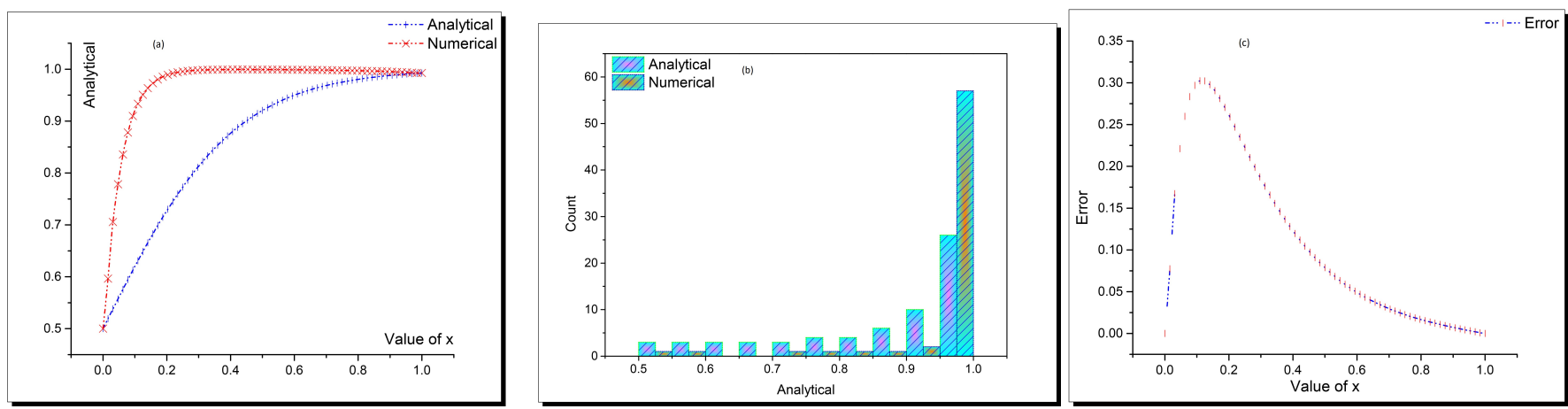

Figure 5. Two-dimensional plot (a), double y-column plot (b) for the matching between obtained analytical and numerical solutions, and two-dimensional plot of the absolute error (c) for Equation (3) with respect to Table 1.
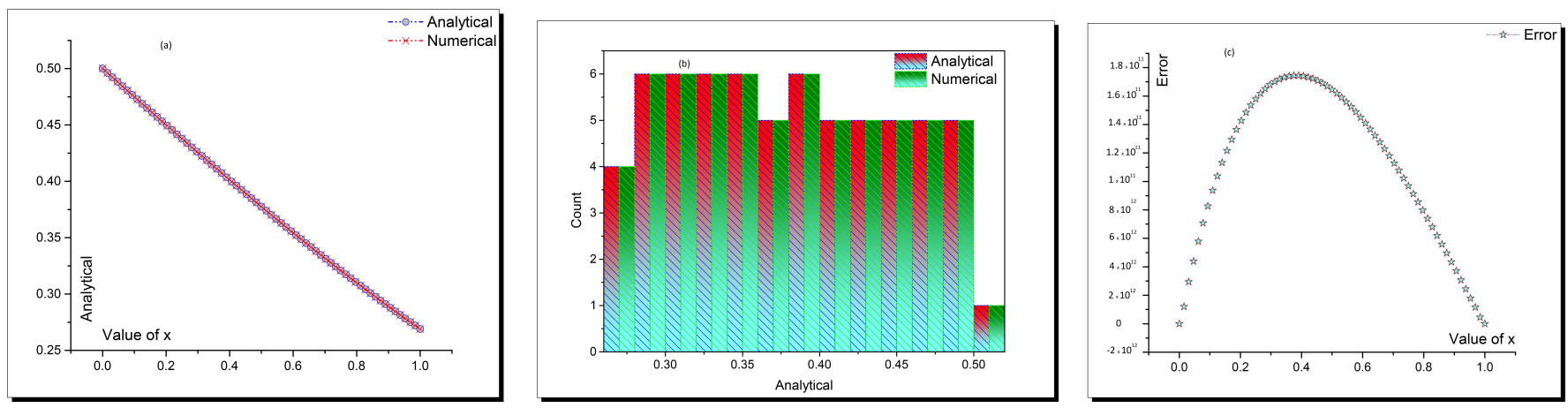

Figure 6. Two-dimensional plot (a) and double y-column plot (b) for the matching between obtained analytical and numerical solutions, and two-dimensional plot of the absolute error (c) for Equation (3) with respect to Table 2. 

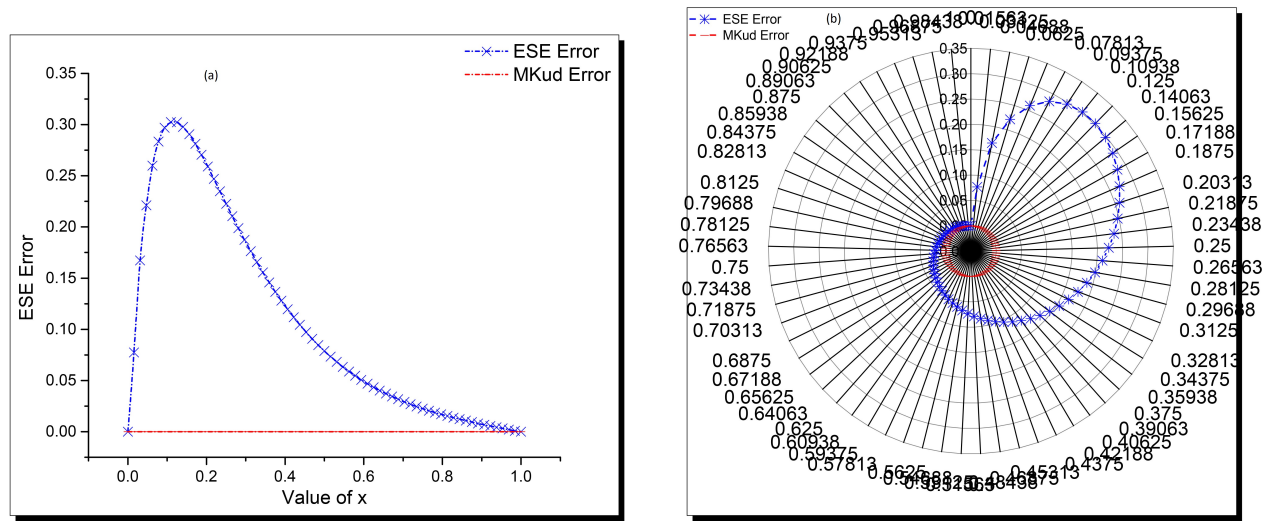

Figure 7. Two-dimensional (a) and radar (b) plots for the calculated absolute error along the MDA, MKud. analytical schemes and the TQBS numerical scheme.

\section{Conclusions}

This research paper has successfully handled the nonlinear (2+1) D-Fisher-KPP model in population genetics and nematic liquid crystals through analytical and numerical investigations. The MDA, MKud., and TQBS schemes have been employed to find accurate novel analytical wave solutions of the considered model. Some obtained solutions have been represented in three different types of sketches. The accuracy of the MKud. method has been verified over the MDA method, where it is our fourth paper in keeping with the title's accuracy on the computational schemes.

Author Contributions: Investigation, M.M.A.K.; Methodology, M.M.A.K.; Software, M.M.A.K. and A.M.A.; Writing—original draft, A.M.A.; Writing—review \& editing, A.M.A. The authors conceived of the study, participated in its design and coordination, drafted the manuscript and participated in the sequence alignment. All authors have read and agreed to the published version of the manuscript.

Funding: This research received no external funding.

Institutional Review Board Statement: Not applicable.

Informed Consent Statement: Not applicable.

Data Availability Statement: The data that support the findings of this study are available from the corresponding author upon reasonable request.

Acknowledgments: Authors want to thank journal stuff (Editors \& Reviewers) for their support and collaboration.

Conflicts of Interest: The authors declare no conflict of interest.

\section{References}

1. Helliar, C.V.; Crawford, L.; Rocca, L.; Teodori, C.; Veneziani, M. Permissionless and permissioned blockchain diffusion. Int. J. Inf. Manag. 2020, 54, 102136. [CrossRef]

2. Axpe, E.; Chan, D.; Offeddu, G.S.; Chang, Y.; Merida, D.; Hernandez, H.L.; Appel, E.A. A multiscale model for solute diffusion in hydrogels. Macromolecules 2019, 52, 6889-6897. [CrossRef]

3. Chandrabose, S.; Chen, K.; Barker, A.J.; Sutton, J.J.; Prasad, S.K.; Zhu, J.; Zhou, J.; Gordon, K.C.; Xie, Z.; Zhan, X.; et al. High exciton diffusion coefficients in fused ring electron acceptor films. J. Am. Chem. Soc. 2019, 141, 6922-6929. [CrossRef]

4. Grebenkov, D.S. Paradigm shift in diffusion-mediated surface phenomena. Phys. Rev. Lett. 2020, 125, 078102. [CrossRef] [PubMed]

5. Iima, M.; Honda, M.; Sigmund, E.E.; Ohno Kishimoto, A.; Kataoka, M.; Togashi, K. Diffusion MRI of the breast: Current status and future directions. J. Magn. Reson. Imaging 2020, 52, 70-90. [CrossRef] [PubMed]

6. Brown, J.L.; Johnston, W.; Delaney, C.; Rajendran, R.; Butcher, J.; Khan, S.; Bradshaw, D.; Ramage, G.; Culshaw, S. Biofilmstimulated epithelium modulates the inflammatory responses in co-cultured immune cells. Sci. Rep. 2019, 9, 1-14. [CrossRef] [PubMed]

7. Hosseininia, M.; Heydari, M. Legendre wavelets for the numerical solution of nonlinear variable-order time fractional 2D reaction-diffusion equation involving Mittag-Leffler non-singular kernel. Chaos Solitons Fractals 2019, 127, 400-407. [CrossRef] 
8. García-Crespo, C.; Soria, M.E.; Gallego, I.; Ávila, A.I.d.; Martínez-González, B.; Vázquez-Sirvent, L.; Gómez, J.; Briones, C.; Gregori, J.; Quer, J.; et al. Dissimilar conservation pattern in hepatitis C virus mutant spectra, consensus sequences, and data banks. J. Clin. Med. 2020, 9, 3450. [CrossRef]

9. Yang, X.; Li, Y.; Zou, L.; Zhu, Z. Role of exosomes in crosstalk between cancer-associated fibroblasts and cancer cells. Front. Oncol. 2019, 9, 356. [CrossRef] [PubMed]

10. Bochner, S. Diffusion equation and stochastic processes. Proc. Natl. Acad. Sci. USA 1949, 35, 368. [CrossRef]

11. Elphick, C.; Coullet, P.; Repaux, D. Nature of spatial chaos. Phys. Rev. Lett 1987, 58, 431-434.

12. Dee, G.; van Saarloos, W. Bistable systems with propagating fronts leading to pattern formation. Phys. Rev. Lett. 1988, 60, 2641. [CrossRef]

13. Van Saarloos, W. Front propagation into unstable states. II. Linear versus nonlinear marginal stability and rate of convergence. Phys. Rev. A 1989, 39, 6367. [CrossRef]

14. El-Hachem, M.; McCue, S.W.; Jin, W.; Du, Y.; Simpson, M.J. Revisiting the Fisher-Kolmogorov-Petrovsky-Piskunov equation to interpret the spreading-extinction dichotomy. Proc. R. Soc. A 2019, 475, 20190378. [CrossRef]

15. Fisher, R.A. The wave of advance of advantageous genes. Ann. Eugen. 1937, 7, 355-369. [CrossRef]

16. Kolmogorov, A.N.; Petrovskii, I.G.; Piskunov, N.S. A study of the diffusion equation with increase in the amount of substance and its application to a biology problem. Bull. Univ. Mosc. Ser. Int. 1937, 1, 1-16.

17. Veeresha, P.; Prakasha, D.; Singh, J.; Khan, I.; Kumar, D. Analytical approach for fractional extended Fisher-Kolmogorov equation with Mittag-Leffler kernel. Adv. Differ. Equ. 2020, 2020, 1-17. [CrossRef]

18. Levchenko, E.A.; Shapovalov, A.V.; Trifonov, A.Y. Symmetries of the Fisher-Kolmogorov-Petrovskii-Piskunov equation with a nonlocal nonlinearity in a semiclassical approximation. J. Math. Anal. Appl. 2012, 395, 716-726. [CrossRef]

19. Luther, R. II. Sitzung am Dienstag, den 22. Mai, vormittags 9 Uhr, im grossen Auditorium des chemischen Laboratoriums der Technischen Hochschule. Räumliche Fortpflanzung chemischer Reaktionen. Z. Elektrochem. Angew. Phys. Chem. 1906, 12, 596-600. [CrossRef]

20. Oruç, Ö. An efficient wavelet collocation method for nonlinear two-space dimensional Fisher-Kolmogorov-Petrovsky-Piscounov equation and two-space dimensional extended Fisher-Kolmogorov equation. Eng. Comput. 2020, 36, 839-856. [CrossRef]

21. Morgado, G.; Nowakowski, B.; Lemarchand, A. Fisher-Kolmogorov-Petrovskii-Piskunov wave front as a sensor of perturbed diffusion in concentrated systems. Phys. Rev. E 2019, 99, 022205. [CrossRef]

22. El-Nabulsi, R.A. Fourth-Order Ginzburg-Landau differential equation a la Fisher-Kolmogorov and quantum aspects of superconductivity. Phys. C Supercond. Its Appl. 2019, 567, 1353545. [CrossRef]

23. El-Nabulsi, R.A. Orbital dynamics satisfying the 4 th-order stationary extended Fisher-Kolmogorov equation. Astrodynamics 2020, 4, 31-39. [CrossRef]

24. Khater, M.M.; Seadawy, A.R.; Lu, D. Bifurcations of solitary wave solutions for (two and three)-dimensional nonlinear partial differential equation in quantum and magnetized plasma by using two different methods. Results Phys. 2018, 9, 142-150. [CrossRef]

25. Khater, M.; Attia, R.A.; Abdel-Aty, A.H.; Abdel-Khalek, S.; Al-Hadeethi, Y.; Lu, D. On the computational and numerical solutions of the transmission of nerve impulses of an excitable system (the neuron system). J. Intell. Fuzzy Syst. 2020, 38, 2603-2610. [CrossRef]

26. Qin, H.; Attia, R.A.; Khater, M.; Lu, D. Ample soliton waves for the crystal lattice formation of the conformable time-fractional $(\mathrm{N}+1)$ Sinh-Gordon equation by the modified Khater method and the Painlevé property. J. Intell. Fuzzy Syst. 2020, 38, $2745-2752$. [CrossRef]

27. Khater, M.M.; Baleanu, D. On abundant new solutions of two fractional complex models. Adv. Differ. Equ. 2020, 2020, 1-14. [CrossRef]

28. Yue, C.; Khater, M.M.; Attia, R.A.; Lu, D. The plethora of explicit solutions of the fractional KS equation through liquid-gas bubbles mix under the thermodynamic conditions via Atangana-Baleanu derivative operator. Adv. Differ. Equ. 2020, 2020, 1-12. [CrossRef]

29. Abdel-Aty, A.H.; Khater, M.M.; Dutta, H.; Bouslimi, J.; Omri, M. Computational solutions of the HIV-1 infection of CD4+ T-cells fractional mathematical model that causes acquired immunodeficiency syndrome (AIDS) with the effect of antiviral drug therapy. Chaos Solitons Fractals 2020, 139, 110092. [CrossRef] [PubMed]

30. Khater, M.M.; Attia, R.A.; Park, C.; Lu, D. On the numerical investigation of the interaction in plasma between (high \& low) frequency of (Langmuir \& ion-acoustic) waves. Results Phys. 2020, 18, 103317.

31. Khater, M.M.; Attia, R.A.; Abdel-Aty, A.H.; Alharbi, W.; Lu, D. Abundant analytical and numerical solutions of the fractional microbiological densities model in bacteria cell as a result of diffusion mechanisms. Chaos Solitons Fractals 2020, 136, 109824. [CrossRef]

32. Abdel-Aty, A.H.; Khater, M.M.; Attia, R.A.; Abdel-Aty, M.; Eleuch, H. On the new explicit solutions of the fractional nonlinear space-time nuclear model. Fractals 2020, 28, 2040035. [CrossRef]

33. Qin, H.; Khater, M.; Attia, R.A. Inelastic Interaction and Blowup New Solutions of Nonlinear and Dispersive Long Gravity Waves. J. Funct. Spaces 2020, 2020, 5362989 . [CrossRef] 
34. Park, C.; Khater, M.M.; Abdel-Aty, A.H.; Attia, R.A.; Rezazadeh, H.; Zidan, A.; Mohamed, A.B. Dynamical analysis of the nonlinear complex fractional emerging telecommunication model with higher-order dispersive cubic-quintic. Alex. Eng. J. 2020, 59, 1425-1433. [CrossRef]

35. Khater, M.M.; Ghanbari, B.; Nisar, K.S.; Kumar, D. Novel exact solutions of the fractional Bogoyavlensky-Konopelchenko equation involving the Atangana-Baleanu-Riemann derivative. Alex. Eng. J. 2020, 59, 2957-2967. [CrossRef]

36. Yue, C.; Lu, D.; Khater, M.M.; Abdel-Aty, A.H.; Alharbi, W.; Attia, R.A. On explicit wave solutions of the fractional nonlinear DSW system via the modified Khater method. Fractals 2020, 28, 2040034. [CrossRef]

37. Abdel-Aty, A.H.; Khater, M.; Attia, R.A.; Eleuch, H. Exact Traveling and Nano-Solitons Wave Solitons of the Ionic Waves Propagating along Microtubules in Living Cells. Mathematics 2020, 8, 697. [CrossRef]

38. Qin, H.; Khater, M.; Attia, R.A. Copious Closed Forms of Solutions for the Fractional Nonlinear Longitudinal Strain Wave Equation in Microstructured Solids. Math. Probl. Eng. 2020, 2020, 3498796. [CrossRef]

39. Demiray, S.T.; Bayrakci, U. Soliton solutions for space-time fractional Heisenberg ferromagnetic spin chain equation by generalized Kudryashov method and modified exp-expansion function method. Rev. Mex. Física 2021, 67, 393-402. [CrossRef]

40. Chu, Y.; Khater, M.M.; Hamed, Y. Diverse novel analytical and semi-analytical wave solutions of the generalized (2+1)-dimensional shallow water waves model. AIP Adv. 2021, 11, 015223. [CrossRef]

41. Shakeel, M. Travelling wave solution of the Fisher-Kolmogorov equation with non-linear diffusion. Appl. Math. 2013 , 4, 35319. [CrossRef] 